\title{
El pensamiento del profesor de Educación Física: un acercamiento a las prácticas educativas institucionales
}

The Physical Education teacher's thought: an approach to institutional educational practices

\author{
Martha Ibette Correa Olarte \\ Nelly Teresa Martinez*
}

Resumen

Este proyecto de investigación de tipo etnográfico pretende develar los pensamientos y creencias que orientan la clase de educación física del licenciado de la Universidad Pedagógica Nacional, UPN, a partir del registro de su discurso y sus acciones pedagógicas en el contexto de la institución educativa escolar de la ciudad de Bogotá de las diferentes tendencias pedagógicas que han permeado su desarrollo. El resultado de la investigación se presenta en cinco categorías de análisis: finalidades, contenidos, metodología, concepto de desarrollo y relaciones maestro-estudiante, elementos constitutivos del modelo pedagógico.

Palabras clave: Pensamiento y creencia del profesor; educación física técnica, educación física Integral, modelo pedagógico.

\section{Abstract}

This ethnographic research aims at disclosing the thoughts and beliefs underlying Physical Education lessons by Physical Education teachers holding a bachelor's degree from the Universidad Pedagógica Nacional. The object of study will be the teachers' record of their discourse and pedagogical actions in the context of school institutions in Bogotá, and of the different pedagogical trends that have permeated his or her development. The research results are shown within five different analyzing categories: goals, contents, methodology, conception of "development", and teacher-student relationships. All of them, elements of any pedagogical model.

Key Words: $\quad$ Teachers' thoughts and beliefs, technical Physical Education, Integral Physical Education, pedagogical model.

Fecha de recepción: 20 de noviembre de 2006

Fecha de aceptación: 4 de diciembre de 2006

1 Profesoras e investigadoras de la Facultad de Educación Física de la Universidad Pedagógica Nacional. 


\section{Antecedentes}

El proyecto se realizó en dos etapas: la primera, en la cual se planteó como proyecto de investigación formativa, surgida del análisis crítico del proceso de formación de licenciados en educación, y la segunda ${ }^{1}$, soportada en los presupuestos de la investigación cualitativa etnográfica bajo una racionalidad crítica.

\section{Introducción}

La necesidad de instaurar prácticas permanentes de reflexión crítica acerca de las dinámicas educativas escolares parte de la hipótesis de que la disposición permanente del maestro permite observar y develar los pensamientos y creencias a partir de los cuales orienta su quehacer, teniendo en cuenta que dichos pensamientos y creencias son construidos desde contextos sociopolíticos, culturales y pedagógicos de la formación y de la experiencia profesional como maestro, y que por tanto su explicitación y comprensión pueden suscitar una resignificación del saber y una adaptación creativa a los contextos cambiantes.

El proyecto intenta develar los pensamientos y creencias de los licenciados a partir del estudio de los aspectos constitutivos del modelo pedagógico, escogidos como categorías de análisis y considerados fundamento histórico-social con el cual las prácticas educativas than estudiado en la escena escolar y que en síntesis corresponden a las preguntas: ¿Qué tipo de persona interesa formar? ¿Cómo o con qué estrategias? ¿A través Je qué contenidos, entrenamientos o experiencias? ¿A ué ritmo de enseñanza debe adelantarse? y ¿Quién y ab́mo se determina o dirige el proceso? Las respuestas a dichas preguntas constituyen las cinco categorías de análisis sobre las cuales se presentarán los resultados de h investigación, que en esencia son: finalidades, contenidos, metodología, concepto de desarrollo y relaciones maestro-estudiante.

El enfoque etnográfico del proyecto permite crear espacios de reflexión crítica y epistemológica de mayor credibilidad, como alternativa para superar la raciomalidad tecnicista de las investigaciones alrededor de - Educación Física, en búsqueda de una racionalidad uritica que dé paso a nuevas formas de pensar lo edu-

Eis esta segunda etapa el proyecto fue aprobado por el CIUP y contó con la participación de diez estudiantes de pregrado del programa de Licenciaura en Educación Física. cativo en general, y la Educación Física en particular, desde donde se propicie una reorganización epistémica de la disciplina.

\section{Objetivos de la investigación}

Como objetivo general se propuso conocer y comprender lo que piensan y creen los licenciados en educación física de la Universidad Pedagógica Nacional sobre su clase; para ello, específicamente se buscó describir e interpretar los pensamientos y creencias que tienen los licenciados de Educación Física sobre el desarrollo de sus clases en relación con las finalidades, los contenidos, las concepciones de desarrollo, los procesos metodológicos y las relaciones maestro-estudiante; de la misma manera, y a partir de la interpretación, se establecieron las relaciones existentes entre el discurso verbal y las realizaciones concretas del maestro inmerso en el proceso educativo.

\section{Categorías de análisis}

Tanto el proceso de recolección de información como su análisis se realizaron teniendo en cuenta las cinco categorías ya señaladas, que constituyen los elementos fundamentales del modelo pedagógico, que históricamente han permitido la comprensión y organización del acto educativo, consideradas en el contexto sociocultural donde ocurre la interacción enseñanza-aprendizaje. En ese sentido y muy sucintamente se conceptualizaron así:

\section{Finalidades}

se entienden como las metas axiológicas, generalmente vinculadas con la ideología predominante, relacionadas con el tipo de persona ideal de cada época particular, los principios generales y categorías universales acerca de las cosas, la lógica, la vida y la sociedad. Reflejan los sistemas de moralidad y normatividad porque son a la vez efecto y síntoma de las categorías que enmarcan la vida intelectual y la circulación de saberes filosóficos de cada época y cada sociedad históricamente determinada.

\section{Contenidos}

corresponden a las decisiones sobre el tipo de información, destrezas, hábitos y conductas que se deben grabar, reforzar o promover en los estudiantes y pueden ser referidos a disciplinas y autores clásicos surgidos de la 
ciencia, y a conocimientos técnicos como códigos y destrezas observables en el desempeño.

\section{Procesos metodológicos}

Constituidos por las estrategias, propuestas, contenidos y métodos con los cuales se aborda el proceso de enseñanza-aprendizaje; se refieren, en suma, a la forma en que ocurren los actos educativos relacionados con los fines.

\section{Concepto de desarrollo}

Es la respuesta al interrogante ¿A qué ritmo debe ocurrir el aprendizaje? y devela el pensamiento que históricamente ha definido al sujeto del proceso educativo especial desde el punto de vista psicológico, pero considerando también factores socioculturales.

\section{Relaciones maestro-estudiante}

Entendidas como la forma en que ocurre el acto educativo y la manera en que los actores participan en las decisiones de la enseñanza. Este aspecto está íntimamente relacionado con la sociología, en particular con las ideologías predominantes y con los modelos políticos imperantes.

\section{Referentes conceptuales}

La fundamentación conceptual del proyecto se desarrolló en dos sentidos; uno referido a los temas básicos que requerían ser clarificados desde el problema de investigación, como: educación, Educación Física, práctica educativa, modelos pedagógicos, pensamientos y creencias y la clase de educación física; y otro, concerniente a la construcción de la metodología etnográfica.

Se aborda la educación como tema central y se concibe como un proceso dinámico que ocurre por etapas durante toda la vida del individuo, con la finalidad de buscar de modo permanente el desarrollo humano, dentro de un contexto social que permita adquirir y renovar los valores significativos para su cultura. La educación debe entenderse y asumirse como una actividad social, que tiene dos funciones fundamentales: mantener la producción científica, conceptual, técnica y artística que ha elaborado la humanidad, y perseguir siempre la formación del sujeto, es decir, el ascenso cada vez mayor a ser humano. Pese a la complejidad de factores que intervienen en la educación del ser humano, es deseable que se busque el desenvolvimiento pleno de las potencialidades humanas en sus dimensiones del pensar, sentir y actuar. En otras palabras, la educación debe apuntar a la integralidad humana, soportada sobre cuatro pilares básicos, que plantea la Unesco: "Aprender a conocer, aprender a hacer, aprender a vivir juntos y aprender a ser" (1996).

La educación física, como parte integral de la educación humana, ejerce una influencia importante en la formación integral del sujeto, razón por la cual ha sido pensada como necesaria y, mucho más aún, indispensable. Ha llegado a considerarse en sí misma como educación integral en tanto ella se ocupa del desarrollo del individuo en sus dimensiones: físicas, psicomotrices, afectivas, cognoscitivas, estéticas y éticas, como lo expresara José María Cagigal, quien considera a la educación física como el más básico modo natural de educar al niño, de una manera globalizada. Planteamiento que a pesar de haberse propuesto en 1979 sigue vigente y es retomado en los textos modernos, como Deporte y ciencia de Juan Rodríguez López (2000), en el cual se refuerza que la educación física es:

la educación a través de su cuerpo, como primera instancia que permanece y acompaña al hombre durante toda su vida... Con el ejercicio integral el hombre se ejercita para capacitarse en todos sus niveles desde la conducta superior hasta las destrezas mecánicas y capacidades sicológicas. Por ello se ha considerado que la educación del cuerpo capacita para construir hábitos y a través de ellos se forja el esfuerzo que es en principio la base de la formación del carácter y el principio del respeto a sí mismo (a su cuerpo) y a los demás... en la instauración de una moral que arranque desde el pacto del hombre consigo mismo, desde sus propias emergencias corporales (84-85).

El sentido de las prácticas puede presentarse en dos direcciones en la medida que estén orientadas por un pensamiento o juicio práctico o por un pensamiento técnico. La primera corresponde al tipo de acción que tiene como propósito la interacción humana, es decir, la relación humana basada en el diálogo, dirigida a la consecución de un fin que no es la producción de un artefacto o cosa, sino a la realización de algún bien moralmente conveniente a la que se concibe como praxis, que implica un trabajo concreto de fabricación que se lleva a cabo según un modelo. De tal modo, la práctica del profesor de Educación Física puede ocurrir en cualquiera de estos dos sentidos, aunque históricamente en su quehacer ha predominado la dimensión técnica. Desde hace algunas décadas se han expresado ideas alrededor de las consecuencias negativas de un enfoque puramente tecnicista de la educación física, y de la ne- 
cesidad de la realización de estudios más profundos que permitan superar dicha condición.

\section{Pensamiento y creencia del profesor}

La fundamentación teórica del proyecto encontró relación clara con la línea de investigación pensamiento y creencia del profesor que adelanta la Facultad de Ciencia y Tecnología de la Universidad Pedagógica Nacional. De tal manera, se asume que el objeto de estudio lo constituyen contenidos de conciencia del docente: sus creencias y pensamientos surgidos de la comprensión que tiene de su propia práctica a partir de sus necesidades, intenciones e intereses como ejes de la realidad.

En tal sentido, se considera que el maestro genera teoría a partir de la reflexión de lo que ha aprendido en la práctica, modificando o confirmando sus propios principios; esto se denomina modelo cíclico de pensamiento del profesor (Anning, 1987). Para Gimeno y Pérez existen dos enfoques a ser tenidos en cuenta para comprender el desarrollo de la investigación sobre el pensamiento del profesor: el enfoque cognitivo, que agrupa todas las preocupaciones de carácter psicológico sobre las operaciones mentales de los profesores en los distintos momentos de su acción pedagógica, y los enfoques alternativos, que se estructuran desde la relación analítica y crítica de principios de la teoría social y de la pedagogía.

En el enfoque cognitivo pueden notarse tres áreas de desarrollo: las investigaciones que centran el problema Zel pensamiento del profesor en la planificación, las que buscan conocer los procesos de razonamiento que ocumen en la mente del profesor durante el desarrollo de su actividad práctica, y las referidas al estudio de las creencias y teorías del profesor, haciendo énfasis en las causas del rendimiento de los alumnos.

Las creencias pueden ser definidas como conocimientos subjetivos poco elaborados, generados a nirel personal para explicar y justificar sus decisiones, actuaciones personales y profesionales y pueden estar dirigidas sobre los actos de enseñar y aprender indistintamente. Autores como Nussbaum y Amartya Sen consideran que las creencias se fundamentan básicamente en la noción de alcanzar decisiones racionales o irraciomales; están basadas en hipótesis desde distintos puntos de vista, que provocan su aceptación o rechazo.

La valoración de las creencias ha variado históricamente. Para el empirismo, las creencias son racionales, uparecen como algo sentido por el espíritu, se discrimi- nan en las ideas, en los juicios y las ficcioneș de la imaginación, se representan por la vivacidad de impresiones sensibles. Para Kant, las creencias son por completo independientes del saber; en el sentido de los racionalistas, que procede por principios reputados supremos, sin previo examen y sin crítica de los límites de la facultad cognoscitiva, la creencia, no como la fe, sino como la razón práctica.

Las personas en sus acciones formulan interpretaciones que se parecen a las creencias por tener un contenido que estructura el modo de ver, pero difieren de ellas en que su función "consiste en orientar, haciendo posible que las personas realicen cambios que se prevén y creen deseables, mientras que la función de las creencias es regir la conducta" (Ibid., p. 30). Si se tiene en cuenta que las creencias son parte del pensamiento del profesor y que son de fuerte arraigo, es preciso considerarlas como determinantes en la constitución del campo profesional de la educación física.

Las creencias se caracterizan por el hecho de requerir consentimiento personal y ser subjetivas. La diferencia con el conocimiento objetivo se mide por el grado de necesidad y aprobación individual por parte del sujeto. Dewey afirma que hay un tipo de pensamiento de las personas, que no es más que el resultado de un consentimiento, de un acto subjetivo. Otro principio de diferenciación es la falta de seguridad respecto al tipo de conocimiento llamado creencia, ya que la seguridad que produce el saber llamado científico es de naturaleza distinta de la producida por el sistema de creencias del maestro en otros ámbitos. Las creencias constituyen principios de acción por medio de los cuales el sujeto es libre para determinar si actúa o no.

A lo largo de la historia se ha identificado la creencia con la fe y se ha opuesto al saber; se ha sustentado que todo saber y, en general, toda afirmación tiene en su base una creencia (Ferrater, 1994). Algunos autores que investigan el pensamiento del maestro oponen la creencia al saber y, en tal sentido, siempre la creencia será un error que al estar directamente relacionado con la acción del maestro hay que corregir para que su práctica mejore y se acerque a la ciencia; todo conocimiento es una creencia, pues todo paradigma es susceptible de ser reorganizado, superado y revolucionado. Dewey afirma que conocimientos que son tomados en una época y por algunos como verdaderos, son posteriormente tomados como erróneos. Desde esta óptica, no hay diferencia fundamental y tajante entre creencia y conocimiento científico; sin embargo, cabe señalar que las creencias corresponden a una lógica diferente de la del conoci- 
miento científico. Como la conducta está regida por las creencias, y éstas tienen un sentido colectivo,

es probable que las creencias de una persona no sólo abarquen las creencias sobre las creencias de las otras, sino también sus intenciones y objetivos, y viceversa. Estas creencias no solo se utilizarán para regir la conducta, sino para modificar las intenciones que las orientan" (Carr).

\section{Diferenciación entre pensamiento y creencia}

Las creencias poseen una carga afectiva y evaluativa que no precisa un consenso social sobre su validez ni sobre su adecuación; son simplemente una aceptación personal. Los pensamientos, por su parte, son un poco más difíciles de encontrar, ya que ellos pueden ser institucionalizados, compartidos o personales, y están asociados con el tipo de conocimiento, la experiencia y las oportunidades prácticas que ofrezca la escuela para la reflexión intelectual. No obstante, los pensamientos de los maestros son el resultado de la interacción e interdependencia de altos contenidos intelectuales, predicativos, creativos y volitivos que permiten la construcción organizada de un pensamiento.

Una síntesis de los aspectos relevantes tanto de los pensamientos como de las creencias señala que las creencias se forman en edad temprana y tienden a permanecer toda la vida, superando las contradicciones causadas por la razón, el tiempo, la escuela y la experiencia. Son las estructuras que soportan el proceso de transmisión cultural, y ayudan a definir y comprender el mundo. Así mismo, el carácter afectivo y evaluativo de las creencias funciona como filtro para interpretar todo nuevo fenómeno. Las creencias educativas deben comprenderse dentro de un sistema cultural y social mucho más amplio; cuanto más antigua es una creencia, mucho más difícil es cambiarla, y en la edad adulta difícilmente se cambia una creencia.

Los pensamientos de los profesores están relacionados con la capacidad de combinar pensamiento organizado, tecnología, currículo, institución, tipo de estudiante, política educativa y nuevos contenidos científicos, entre otros aspectos; la edad es un factor determinante en la resistencia al cambio de algunos agentes educativos, como la autosuficiencia y las oportunidades que brinda la escuela para mantenerse abierto y dispuesto a las transformaciones educativas. Para concretar, los pensamientos de los docentes pueden estructurarse de acuerdo con tres tipologías de conocimiento: proposicional, estratégico y de casos.

\section{Metodología}

Esta investigación se realizó bajo una perspectiva etnográfica, entendida como la descripción de hechos sociales e institucionales y como una forma de estudiar y comprender la vida humana (Tezanos, 1998), referida en este caso al espacio inmediato donde el profesor de Educación Física pone en acción en forma más global sus pensamientos, valoraciones, conceptualizaciones, creencias, actitudes, sentimientos, habilidades y demás componentes de la educación. Los resultados permitieron una reconstrucción analítica del espacio de la cultura escolar llamada clase de Educación Física intentando establecer algunas relaciones entre el pensamiento y el actuar del profesor en relación con las finalidades, los contenidos, las actividades del proceso metodológico, las concepciones de desarrollo y las relaciones maestroestudiante que el profesor atribuye, piensa o cree adecuados dentro del espacio de la clase.

\section{Fases de investigación}

La primera etapa del diseño del proyecto se desarrolló en el marco de la investigación formativa y de la investigación formal, en La Facultad de Educación Física de la UPN. Puede verse información detallada de dicho proceso en un artículo publicado en la revista Lúdica Pedagógica $\mathrm{N}^{\circ} 8$, que presenta una síntesis del problema y el proceso de construcción de las preguntas y categorías de análisis de la investigación antes descritas. Este artículo se ocupa de la etapa de sistematización, análisis e interpretación realizada siguiendo las sugerencias de la investigadora Araceli de Tezanos en Una etnografía de la etnografía (1988), que propone dos fases: una descriptiva y otra interpretativa. La fase descriptiva consistió en conocer y descubrir qué es y cómo es el objeto de estudio, a partir de la información obtenida en los diferentes tipos de registro, tal cual se tomaron durante la recolección ${ }^{2}$, y comprendió: lectura en escucha: lectura, organización, agrupación de registros y verificación; elaboración de subcategorías para establecer semejanzas y diferencias teniendo en cuenta los hechos similares, recurrentes y casos excepcionales. Por último, producción de texto descriptivo a partir de la triangulación: ordenación-verificación-escritura de texto descriptivo; el resultado de esta fase fue la cons-

\footnotetext{
2 Se recolectó información por medio de tres instrumentos: cuestionario, entrevistas y fichas de observación.
} 
trucción de textos con sentido y significado respetando la voz del otro. La fase de interpretación. Consistió en el descubrimiento de las relaciones que dan sentido y existencia a la clase de Educación Física como objeto de estudio y permitió ampliar su comprensión a partir de la interpretación y la hermenéutica. En esta fase se realizó una triangulación entre las descripciones elaboradas en el proyecto, las teorías existentes y el conocimiento de los investigadores. Como lo señala Tezanos, corresponde a la creación de interpretaciones acerca de los pensamientos de los profesores y a la comprensión de los fenómenos más significativos a partir de los cuales se elaboraron los textos finales con pretensión de teorización inicial.

\section{Población y muestra}

Para el desarrollo de la fase de aplicación definitiva de instrumentos se contó con la colaboración de 19 licenciados en Educación Física, egresados de la Universidad Pedagógica Nacional, que trabajan en instituciones educativas de las localidades de Kennedy, Barrios Unidos y Bosa.

\section{Resultados}

La siguiente es una síntesis de los textos interpretativos construidos a partir de los textos descriptivos elaborados en la primera fase. Hacen relación a las cinco categorías de análisis del proyecto de investigación y sus respectivas subcategorías y son el resultado de un segundo nivel teorización ${ }^{3}$ y reconstrucción del objeto de estudio: La clase de Educación Física. Dichos textos develan las paradojas entre el discurso verbal del profesor, expresado en los cuestionarios y las entrevistas, y las acciones realizadas en las clases. Sobre dichos textos volveremos más adelante, una vez sean sometidos a la reflexión crítica y sistemática de manera que puedan darse a conocer en una publicación de mayor amplitud.

\section{Pensamiento sobre las finalidades}

Desde esta categoría se abordaron el pensamiento y las creencias del profesor como dimensión teleológica que evidencia los propósitos fundamentales de la

El primer nivel lo constituyen los textos descriptivos explicados y contenidos en la primera fase de la investigación. Dichos textos corresponden a la sistematización de trozos de registro, que dan cuenta del discurso y las acciones del maestro de una manera anecdótica y particular y no se presentan en este artículo. clase de Educación Física. En este sentido, se halló, en primer lugar, una finalidad físico-biológica, en segundo lugar, una preocupación por el manejo del tiempo libre, mientras que al desarrollo de valores y la formación del pensamiento se les otorga un tercer y un cuarto lugar, respectivamente; en última instancia, y dentro de los propósitos buscados de manera intencional por el maestro, se encuentra la preocupación por la formación integral del estudiante. Paradójicamente, el $90 \%$ de los profesores expresan en forma verbal que el enfoque de su clase es de educación física integral, y sólo un $10 \%$ considera y acepta que su clase es tecnicista. De tal manera, y como se verá más adelante en el texto interpretativo, la afirmación de integralidad de la clase de Educación Física queda en entredicho. En síntesis, de la categoría Finalidades se encontraron las subcategorías (que se relacionan en el cuadro 1. A continuación se presenta, a manera de ejemplo, un aparte de uno de los textos descriptivos, surgidos de las entrevistas subcategoría Educación Física... Educación Integral.

\section{Educación Física... Educación Integral...}

La Educación Física, desde la parte motriz, puede aportar a las demás dimensiones del ser holístico... es una competencia primordial donde se debe trabajar la parte axiológica o de formación de valores..." (E7: 4) ${ }^{4}$, “... es la materia más importante para enseñar valores, enseñar la parte de lealtad, la parte de sinceridad, la parte de honestidad, la parte de responsabilidad, la parte de quererse a sí mismo, la parte de la lucha por el bienestar de sí mismo” (E1: 3), “... es la clase más completa, el alumno aprende conocimientos y habilidades...".

(E5: 7) además “.... aporta mucho, ya que desde la parte motriz se puede trabajar y aportar a las demás dimensiones del ser holístico... la parte axiológica o de formación de valores, que en este caso enseñan a través del juego, como las normas sobre el respeto, la honestidad y la parte comunicativa". Permite también "la formación de valores.... en específico, la autoestima, la seguridad y la confianza y el respeto a los demás..." (E5: 3), por ejemplo, "... en un juego... lo importante es hacer las cosas bien, llegar, así sea de último, no ganar pasando por encima de sus compañeritas... de las otras niñas" (E5: 2).

\footnotetext{
4 La información entre paréntesis indica el número de la entrevista y la página de donde proviene el registro. En este caso Entrevista 7, p. 4.
} 


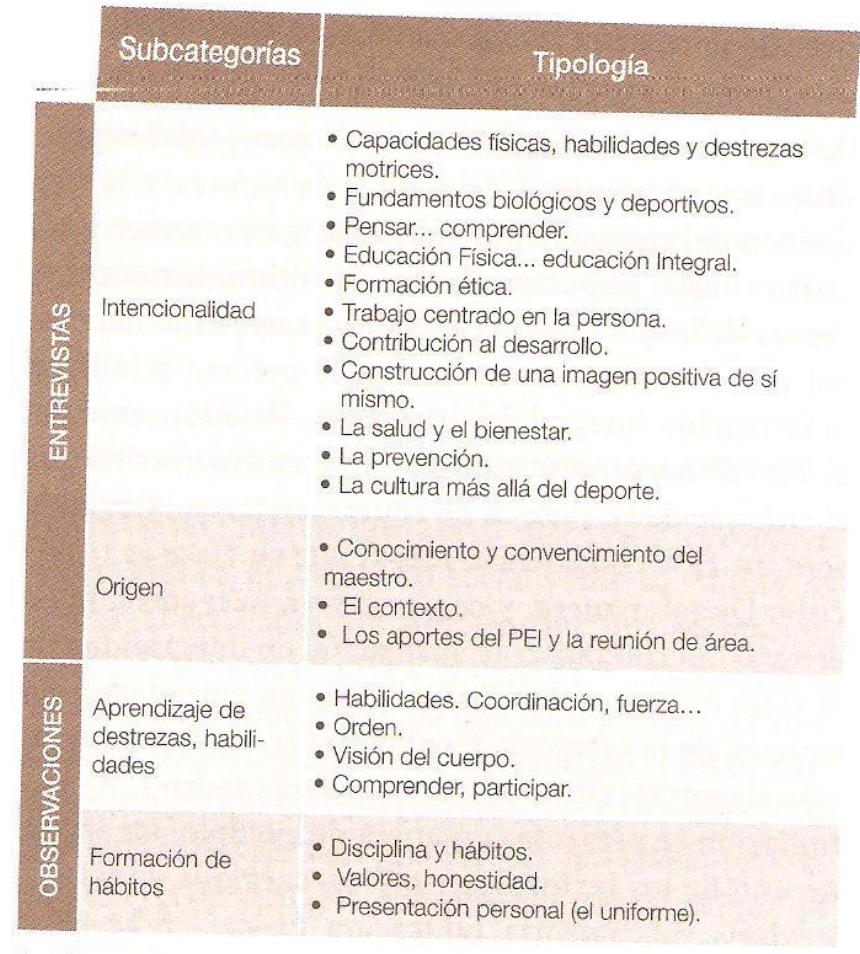

Cuadro 1. Subcategorías de la categoría Finalidades.

Las finalidades de la clase de Educación Física en el contexto de los elementos que conforman el modelo pedagógico representan el fin último de todo el proceso educativo escolar. Están representadas por los valores que se espera formar en el individuo una vez ingrese al sistema educativo, y se constituyen en el reflejo de las necesidades y tendencias de nuestra sociedad a partir de la consideración de factores individuales y de los fenómenos socioculturales en los cuales se insertan la vida escolar, la actividad recreativa, deportiva y de actividad física y, por supuesto el individuo en su totalidad. En este sentido, las finalidades de la clase, en el pensamiento del profesor, responden, en primer lugar, a la comprensión que se tiene sobre la organización y función de la vida escolar y académica y su relación con la sociedad en general $y$, en segundo lugar, se derivan del sistema de creencias que el maestro ha construido a través de su historia de vida y de su visión de hombre y cultura y las relaciones que deben mediar entre ellos.

De la comprensión que se tiene de la vida escolar surgen enunciados sobre finalidades que aparecen como generalidades dentro de la escuela, y que históricamente van transformándose al vaivén de las tendencias educativas impuestas por la ley o son promovidas por movimientos sociales locales; entre ellas se encuentran: la búsqueda de la educación integral del individuo, el desarrollo de todo su potencial y la comprensión y reconocimiento de la necesidad de promover el desarrollo de las diferentes dimensiones que integran al ser humano.

Dichas finalidades construidas, compartidas y aceptadas a través de los proyectos educativos institucionales (PEI), cumplen una función reguladora en la escuela, y se espera que tengan el mismo efecto en la sociedad. Por eso aparecen como el fin último del proceso escolar: educar para vivir en sociedad, para integrarse de manera positiva y productiva al sistema de valores sociales imperante. Dichas finalidades reflejan las necesidades y temores sociales frente a la formación de los jóvenes; por ello, tanto la escuela como el maestro enfatizan en su obligatorio cumplimiento. Así, igual que propone una labor de convencimiento respecto a la conveniencia de aceptarlas como forma de vida, también construye e impone un sistema sancionatorio, que castiga a aquellos individuos que no se comportan de acuerdo con sus enunciados. Ante este grupo de finalidades el maestro de Educación Física actúa dentro del marco de procedimientos descritos en el Manual de Convivencia y se apoya en otras instancias escolares para complementar su función de educar aquellos jóvenes que así lo requieran. En ese momento abandona su función educadora, porque prefiere dedicar el tiempo escolar al desarrollo de su clase particular.

De esta manera, la institución educativa, y dentro de ella la clase de Educación Física, cumple una función social muy particular, pues las finalidades que se proponen institucionalmente surgen de una lectura particular del contexto social, cultural y geográfico en el cual se encuentra inmersa la escuela. Es decir, dichas finalidades tienen que ver con el tipo de comunidad y con el pensamiento que los maestros y directivos expresan de los fenómenos que ocurren en su interior y de la reflexión crítica que los actores hacen de ella. Frente a estas finalidades surgidas de la interpretación del contexto escolar el maestro asume una postura muy firme desde el discurso, pero muy tangencial desde la práctica, ya que en la medida que reconoce y piensa en el estudiante como una totalidad decide al mismo tiempo no involucrar ni encaminar los temas específicos de la clase de Educación Física a la búsqueda de dichas finalidades.

Existe otro grupo de finalidades que el maestro de Educación Física considera específicas de su clase. Frente a ellas, reconoce una total responsabilidad social personal; son aquellas que se centran en el antiguo lema griego: cuerpo sano-mente sana, un tanto oculto baic los enunciados de progreso y avance científico. En est sentido, las finalidades refuerzan el postulado del ejercicio y la actividad física como agente mediador y factor 
= reconocimiento social. La clase de Educación Física es plataforma que promueve en los individuos la necesidad de transformar su estilo de vida: de la pasividad a la actividad; pasividad entendida como inmovilidad, enfermedad, carencia, fealdad, obesidad y fracaso; y actividad, como parte del modelo de deber ser individual r social, que se asocia con bienestar, salud, aceptación, belleza, éxito y triunfo.

Este discurso constituye el énfasis del pensamiento del maestro sobre la clase de Educación Física y parte de la consideración del individuo-estudiante como un ser dual, en un sentido, caracterizado por la carencia y, en otro sentido, lleno de problemas que debe resolver a partir del conocimiento que adquiere en la escuela - en particular en la clase de Educación Física. Este discurso bien podría enunciarse como una teoría de Ia ausencia. Es decir, el maestro considera a sus estudiantes como seres vacíos sobre los cuales debe actuar como protagonista; para ello, se apoya en el discurso actual sobre los estilos de vida y encuentra respaldo en la información de los medios masivos de comunicación y en la creciente valoración del cuerpo como medio y fin de la realización personal y profesional. De tal manera y en coherencia con su posición frente al estudiante, el maestro asume el papel de un mesías, dueño de las preguntas y sabedor de las respuestas y de los medios para alcanzar los cambios deseados en los estudiantes; y los estudiantes continúan en el papel de receptores pasivos, que aprueban y repiten la multiplicidad de experiencias propuestas por el maestro, seguros de que ellas los conducirán a una nueva y mejor forma de vivir.

Así pues, el maestro y la clase de Educación Física se constituyen en la fuente donde el estudiante puede satisfacer las necesidades de acción que lo llevarán al reconocimiento y la aceptación social, bajo la condición de seguir un camino que incluye ante todo un proceso de autoconocimiento, dominio y control corporal a partir del hacer: hacer ejercicio, hacer deporte, hacer repeticiones. Únicamente la acción valida el proceso de formación del individuo, como medio y fin del proceso educativo escolar, considerándolo como deber ser de la educación física y como posibilidad de alcanzar el ideal de hombres impetuosos, fuertes, emprendedores, persistentes y viriles y de mujeres fuertes, bellas, capaces y triunfadoras, ambos estilos de vida premiados por la sociedad de consumo.

Otras creencias culturalmente aceptadas hacen parte del fundamento sobre el cual se construye el pensamiento del maestro en torno a las finalidades de la clase de Educación Física. Es el caso de la premisa: Deporte es salud, salud del individuo y salud pública; la práctica de actividad física, ya sea en la modalidad de práctica deportiva, actividad recreativa o como ejercicio, se considera antídoto contra los flagelos modernos que atacan la a sociedad, llámense ellos delincuencia, adicción, falta de identidad o enfermedades silenciosas tales como infartos, derrames, diabetes, cáncer y hasta sida. El maestro piensa que la Educación Física cumple un papel primordial y considera su deber promoverlo dentro de la clase, ya sea como factor de prevención o como solución definitiva.

Frente a este panorama, puede afirmarse que el pensamiento del maestro en torno a la clase de Educación Física en la escuela es causa y efecto, promotor y resultado, de una serie de pensamientos que históricamente los han permeado, pero frente a los cuales no ha dedicado un proceso de reflexión consciente. Por tanto, la clase y el maestro aparecen como elementos repetidores de viejos esquemas, moldeados de manera superficial por los rezagos de algunos movimientos sociales y pedagógicos que han tenido repercusión en la sociedad. El maestro conserva la idea de que se enseña a través de la disciplina consciente, basada en el orden y el respeto mutuo, en el trabajo de campo, siempre en pro de un bienestar común. Estas ideas, propuestas por Antón Makarenko y aprendidas por el maestro en su etapa de formación, lo llevan a actuar como modelo y guía, como padre de niños sin padre y como compañero y amigo, pero con muy poca conciencia acerca de su origen y de sus implicaciones.

La idea del mejor esfuerzo y el sacrificio es también una clara evocación de la vieja tradición cristiana del sufrimiento ahora, para obtener una vida eterna en el más allá. Se debe sufrir en la clase, sudar, alcanzar el máximo rendimiento, para construir los hábitos necesarios con los cuales el individuo alcanzará, en el futuro, una mejor forma de vida fuera del sistema educativo formal, ya que será una mejor persona. El maestro repite el valor de la acción; porque no tiene dudas, tiene certezas sobre su hacer fundamentado en la acción; cree en él y en sus planteamientos, y considera que ya todo está dicho, hecho y terminado. Se adhiere a ese saber como a un feudo; parece que no puede imaginar otro camino que no sea el de la acción y el sacrificio, y un tanto ciego por sus taras, quiere incluir a sus estudiantes en las prácticas deportivas civilizadas del aeróbico, el esfuerzo físico y el sudor, en detrimento de lo lúdico, del juego libre, de la emancipación de la sensibilidad fuera de lo culturalmente establecido. 
Paradójicamente, el maestro expresa de manera verbal la influencia que ha recibido de ciertas corrientes pedagógicas de tipo mas crítico y liberador, como la pedagogía de Paulo Freire, desde donde argumenta que la mejor manera de aprender es liberándose del influjo de los paradigmas que nos oprimen y que éstos se rompen cuando el estudiante accede al poder que se adquiere gracias al conocimiento. En estos casos, el diálogo y la palabra son tenidos en cuenta como factores de transformación de los educandos, casi nunca se involucra el cambio del maestro; el maestro hace referencia a la reflexión del individuo y su concientización, en especial y casi particularmente acerca de sus carencias, como única vía para que se libere y pueda trascender su realidad. Sin embargo, es el maestro, desde sus pensamientos, quien evidencia las crisis en el individuo, es él quien conoce sus carencias y es a través de él como el estudiante se da cuenta de su situación y toma las acciones que le permiten liberarse de estas carencias. Es el maestro quien las define y categoriza y es el mismo maestro quien le señala el camino por donde alcanzará ese nuevo estado de libertad. Esta intención emancipadora del maestro de Educación Física se desdibuja en la acción, ya que en la realización de la clase, una vez más, elige el camino fácil de ser protagonista y centro de la acción educativa que vela por sus educandos y por su adaptación dentro de la sociedad y el deber ser.

En la acción cotidiana de la clase, el maestro de Educación Física se esfuerza por inculcar a sus estudiantes la idea del deber ser, siempre asociada a ser como él, a hacer lo que él mismo hace o hizo, a que repita esquemas a partir de lo que él, como maestro sabedor, distribuye y prodiga. En ese sentido, no se adivina en el maestro una intención emancipadora; por el contrario, es evidente una intención de compartir con él las cadenas que lo esclavizan y mantienen inerte en la sociedad. El discurso acrítico del maestro frente a los fenómenos sociales y en particular frente a la Educación Física lo convierten en un maestro descontextualizado, fuera del tiempo y del mismo movimiento que tanto promueve. Así, salta con facilidad de unas finalidades a otras sin profundizar en ninguna; al parecer no distingue entre pensar, profundizar y hacer las praxis dentro de su clase. Se queda en hacer prospecciones de sus actos y abandona la tarea de saber por qué y para qué, limita su saber al cómo, que a modo de oración repite día tras día en su clase a partir de la misma rutina.

En esta perspectiva, el pensamiento del maestro acerca de las finalidades de clase de Educación Física parece estar suspendido en el tiempo y paradójicamente refleja una preocupante pasividad histórica, en la cual la reflexión y la autocrítica no tienen cabida. Vale la pena preguntarse entonces: ¿Hacia dónde nos conduce la búsqueda de dichas finalidades, que poco o nada tiene que ver con la acción reflexiva del maestro? ¿Qué consecuencias trae consigo la falta de reflexión crítica del maestro dentro de la escuela? Es importante llamar la atención hacia la necesidad de dudar; en tanto que la certeza que acompaña la acción del maestro dentro de su clase es la misma que no le permite dudar y, por tanto, cambiar, no es posible dudar de aquello que, en primer lugar el maestro no ha creado; simplemente ha repetido a través del tiempo. Los valores y conocimientos que el maestro enuncia y promueve desde el discurso y desde las actividades de la clase han existido desde siempre y se han posicionado con cierta comodidad dentro de un sistema de valores sociales sobre los cuales es incapaz de reflexionar para hacerlos evidentes como parte de su labor educativa dentro de la clase misma. Únicamente la duda ha permitido al maestro sustituir de manera parcial o definitiva el entramado de creencias sobre las cuales fundamenta su acción educativa.

\section{Pensamiento acerca de los contenidos de la clase de Educación Física}

\section{Clasificación inicial}

El qué enseñar en la clase de Educación Física en el pensamiento del maestro está constituido por varios tipos de saberes, los cuales son asumidos con diferentes sentidos y significados y podrían clasificarse en: contenidos de primer orden, relacionados con el saber cómo, el saber función y el saber hacer. Los primeros se refieren a globalidades culturales tradicionalmente aceptadas, como el deporte, la gimnasia y la danza. Incluyen conocimientos en torno al saber técnico disciplinar pero pueden pensarse como teóricos, y en tal caso se relacionan con carencias biológicas, con la historia del deporte y de la actividad física, la fisiología del ejercicio y la administración deportiva. El saber función y el saber cómo se piensan como saberes establecidos, y lo que define su presencialidad o no como objetos de aprendizaje y enseñanza dentro del programa o currículo de Educación Física está sujeto a decisiones externas al maestro, como los factores normativos de orden superior central (políticas de Estado o directrices institucionales), la situación de los estudiantes, las tendencias de las disciplinas normativas (cómo la pedagogía, la didáctica y la fisiología del ejercicio, la gradación escolar y el desarrollo 
biológico del estudiante). El saber hacer y el saber cómo no forman parte del contenido programático de la Educación Física porque son saberes en los que el ambiente físico y el objeto hacen parte del saber; es decir, que su enseñabilidad tiene determinantes en el ambiente que deben ser tenidos en cuenta por el maestro.

Los pensamientos de segundo orden se caracterizan por ser menos explícitos. Requieren un uso mínimo de lenguaje explicativo y más de la enseñanza tácita, y se refieren a la adquisición de hábitos de comportamiento frente a la autoridad, la disciplina, el orden, la obediencia, el comportamiento social, en particular el trabajo en parejas o grupos, las sanciones, la evaluación, el control del comportamiento, la discriminación de los no competentes o integración entre géneros. Además, sin hacer una diferenciación categorial fundamentada, es posible incluir otros tipos de saber en el pensamiento del maestro: Haceres y saberes importantes: se exigen, se controlan y evalúan y, en consecuencia, deben aprenderse y corresponden a las destrezas, al rendimiento y a la Educación Física. Haceres y saberes secundarios: se usan para crear ambiente, descansar, tienen la libertad de ejecución por parte del estudiante, no se evalúan ni controlan y suelen hacerse al final de las sesiones de clase. Saberes de carácter instrumental: se refieren a la actividad física como medio para otros propósitos formativos no inherentes a la actividad corporal. Saberes $y$ haceres inherentes al objeto y al medio: En los cuales el espacio donde se realiza y el objeto con que se realiza deben estar en armonía, la ausencia de uno de ellos no permite su enseñanza. Y los saberes permanentes, invariantes: se consideran fijos, porque hacen parte de una rutina y pueden ser realizados por los estudiantes sin una orientación específica. Por último, los pensamientos de tercer orden están referidos a las acciones corporales mediante las cuales pueden realizarse los dos saberes anteriores, y comprenden movimientos básicos y rudimentarios.

La metáfora del trabajo

\section{en relación con los contenidos}

El profesor de Educación Física, al referirse al desarrollo de los contenidos y actividades de la clase, emplea los términos trabajar y trabajo, con un sentido de esfuerzo, dedicación y sufrimiento e intensidad. A ambos términos se les asignan varios significados. Un primer sentido invoca las acciones encaminadas a lograr actuación o ejercitación de la condición social y moral del estudianrepresenta una acción con una finalidad formativa.
Una segunda acepción implica la ejercitación del cuerpo, de sus cualidades y potencialidades corporales orgánicas. Una tercera acepción se relaciona con lo que se ha planificado, lo que se debe llevar a la práctica, una temática o unidad de acción. Un cuarto sentido, enunciado como nombre o sustantivo, se relaciona directamente con los contenidos programáticos de la Educación Física; y un último sentido se dirige a la enunciación de las actividades globales de un periodo académico, de un año o de toda la educación primaria o secundaria. Por los contextos en que el maestro usa el término, puede establecerse que existe una ambigüedad conceptual y didáctica manifiesta en una imprecisión y una ausencia de terminología técnica que exprese con más certeza el sentido de sus pensamientos. Quizá lo que los términos trabajar y trabajo ocultan sea el verdadero sentido de la intervención del maestro, las relaciones de poder entre él y el estudiante y entre él y la temática. En conclusión, puede afirmarse que el uso del término trabajar en tales condiciones es reduccionista, evasivo y polisémico.

\section{La planificación, la programación \\ y el curriculo de la Educación Física en el pensamiento del profesor}

La organización de los contenidos se presenta en el pensamiento del maestro como unidad indisoluble manifiesta bajo los términos de planeación, programación y currículo, acciones a las cuales se refiere indistintamente como funciones y como creencias morales que le permiten saber hacia dónde orientar su quehacer diario, o del año.

A la planificación, la programación y el currículo, como acciones derivadas del deber ser de la docencia, el maestro añade la preparación de o para la clase, frente a la cual existe un pensamiento psicológico, bien sea positivo, como condición facilitadora para afrontar dinámicas difíciles de los grupos escolares, o como una situación que problematiza el quehacer docente. Estandarizar y establecer una forma mecánica de proceder frente a la enseñanza es visto como un ideal de eficiencia, lo que sin duda tiene un asiento en la racionalidad técnica del docente de educación física.

\section{El pensamiento del maestro frente al cambio}

En el maestro de Educación Física existen pensamientos relacionados con el cambio de contenidos, de enfoques metodológicos o de evaluación. La introducción de innovaciones educativas o disciplinarias se expresan con la presencia o introducción de términos sencillos 
a los cuales por lo general se refiere fuera de clase y esporádicamente dentro de las interacciones con los estudiantes, sin que medie una explicación sobre ellos, para buscar su apropiación en el grupo. Esta situación genera en el estudiante confusión y desubicación. Sin embargo, espera y exige a los estudiantes la comprensión de los nuevos términos con un carácter de obligatoriedad, pero ante los cuales el maestro no asume compromiso alguno, cree que no es su tarea, ya que el logro de nuevas comprensiones es obligación del aprendiz. Esta obligatoriedad de tener un saber previo en el estudiante es una creencia permanente en el docente; el deber y el tener un saber previo o una condición motriz son condicionantes para el éxito de su enseñanza, de su función como maestro, pero el papel que juega el docente frente a estos "deberes" de la formación no son pensados como parte del quehacer educativo, específico del profesor de Educación Física, ni de la didáctica del área.

\section{El juego como contenido especial}

Sobre el juego, dentro de la categoría hacer por hacer, el profesor tiene un pensamiento paradójico. De un lado, lo discrimina y subvalora, y de otro, lo reconoce como una dinámica libertaria de la Educación Física. Su importancia dentro de la clase se ubica en jerarquía secundaria de poca exigencia corporal, frente a la cual el maestro no requiere aportar. El juego se muestra en abierta oposición al contenido programado y planeado para la clase; por tanto, no tiene implicaciones corporales que conllevan rigor, repetición, control, secuencia, atención selectiva del profesor y del estudiante, mayor dedicación en tiempo, en observación y corrección. Otra forma de pensar el juego como saber instrumental le atribuye utilidad para adquirir aprendizajes que no son específicos de la Educación Física, pero que son necesarios para la formación del joven. Lo interesante es que lo considera como el espacio de libertad para el estudiante y para él como maestro. El juego puede ser pensado como mediación didáctica para el aprendizaje de un objetivo técnico y en tal caso tiene reglas que requieren la dirección del docente, su seguimiento y su evaluación. Otra mirada frente al juego en el pensamiento del maestro se refiere al juego como sentido lúdico, cultural. $\mathrm{Al}$ respecto, entonces lo concibe por fuera de la programación de la Educación Física y constituye junto con la danza un campo de actividad básicamente cultural, de realización personal auténtica del estudiante. Ante tanta ambigüedad cabe preguntarse si existe en el pensamiento del docente claridad conceptual sobre el juego.

\section{Pensamiento sobre el método}

Una mirada clasificatoria del pensamiento del maestro sobre el método muestra que éste, al referirse al método, los procedimientos y las técnicas para la enseñanza, expresa conocimientos o saberes sobre: el método en la enseñanza de la Educación Física y sobre los principios y formas que guían la enseñanza y el aprendizaje.

\section{Saberes sobre el método de enseñanza de la Educación Física}

\section{El método: ¿cuestión de enunciado?}

El pensamiento del maestro en relación con el método para la enseñanza de la Educación Física coincide con las denominaciones empleadas en el pensamiento científico de tipo empírico-analítico de las ciencias naturales. Por ello, la inducción y la deducción, el análisis y la síntesis aparecen ligados al proceso de conocimiento disciplinar centrado en saberes esencialmente deportivos y gimnásticos. Estos enunciados sobre los métodos específicos para la enseñanza de la Educación Física se soportan en la creencia de que son específicos para la enseñanza en el área, se muestran como incuestionables, obvios y connaturales. Su conceptualización o entendimiento ocurre mediante la ejemplificación de saberes del campo deportivo y a partir de términos cotidianos y comparaciones. Lo interesante de este pensamiento es que el método adquiere un sentido genérico y específico al mismo tiempo. Genérico porque se enuncia como verdades universales y siempre existentes dentro del área; y específico porque el docente afirma emplearlos de una forma distinta y singular para enseñar los diferentes campos de saber. En esta concepción, el método aparece ligado al campo de saber, lo cual demuestra que el maestro no logra precisar ni articular conceptualmente los procedimientos de enseñanza en su aspecto operativo, diferenciándolos del contenido específico.

Los métodos de enseñanza de la Educación Física se enuncian como invariables en el tiempo. Se nombran, pero no se describen, se perciben como interiorizados y en estrecha relación con el saber específico; sin embargo, en la acción no se evidencia la diferencia entre la deducción y la inducción. El método es en la acción una unidad invariable que va de las partes al todo y que pone en juego la relación maestro-saber específico de la Educación Física-estudiante, en las denominadas sesiones de la "clase". El método es visto en la clase como un conjunto de acciones y actividades que giran alrededor de intencionalidades en una dinámica de tres momentos 
interdependientes e interrelacionados: iniciación, nudo o parte central, y declinación o parte final. Esta organización y sentido de la acción docente podría equipararse con el proceso biológico: nacimiento-crecimiento y desamollo, y declinación-muerte terminación.

La primera fase, iniciación, prepara el cuerpo para un momento significativo del aprendizaje motriz, su intencionalidad está relacionada con la adquisición de un patrón o modelo de cuerpo, en cuatro sentidos: cuerpo presente, contable e identificable; cuerpo mismidad, uniforme; cuerpo obediente, alineado, motivado; cuerpo dispuesto al esfuerzo, al trabajo, la exigencia, el rendimiento, el éxito. La segunda fase de la sesión de clase, nudo o parte central, evidencia el saber enseñar lo específico de la disciplina y el deber aprender lo que se tiene que hacer. Corresponde al tema y evidencia su concepción de saber disciplinar, es esta fase la que el profesor concibe como de aprendizaje y adquisición de la destreza motriz ligada a unos principios de aprendizaje amparados en la repetición y la demostración (evidencia empírica)

La tercera fase de la clase es pensada independientemente de las dos anteriores y se hace para regresar al estado fisiológico inicial. Incluye otras intencionalidades formativas generales relacionadas con el comportamiento moral y disciplinar del estudiante. Se permiten estados de deliberación motriz y emocional a través de la inclusión de actividades lúdicas. Como proceso, esta fase no tiene importancia para el docente y no implica ninguna secuencia ordenada.

En el pensamiento del profesor de Educación Física adquiere especial importancia el contexto desde la dimensión social, entendida como la presencia o la ausencia de "compañeros" para realizar el proceso de aprendizaje. Esta creencia refleja el imaginario del deporte como medio socializante, donde el compañero cumple roles de apoyo, como observador, evaluador, copartícipe de la tarea de movimiento o de optimización de recursos y materiales didácticos, e incluso de orientador del aprendizaje. En la misma dimensión social el maestro piensa que el estudiante debe aprender ciertas rutinas necesarias e indispensables, porque presume que la repetición de un esquema o forma organizativa garantizará el aprendizaje de un hábito. Este tipo de prácticas y pensamientos evidencian los mecanismos de inmovilidad de la Educación Física tanto en el saber cómo como en el saber qué, de donde puede deducirse un pensamiento sobre la enseñanza basado en una concepción de saber inmutable, una concepción de desarrollo uniforme establecido básicamente desde la dimensión bio- lógica, que no es afectado por las condiciones sociales del entorno inmediato.

\section{Principios que guían \\ la enseñanza y el aprendizaje}

El maestro piensa y cree sobre el aprendizaje que es hacer, ejecutar la acción, vivirla, sentirla, mostrarla para ser verificada. La concepción de aprendizaje implica la repetición hasta la estabilización o hasta el agotamiento físico, la permanencia en el tiempo y la superación del momento mismo de la adquisición. Para lograr ese conocimiento sobre el saber cómo se requiere una desarticulación de la globalidad en elementos más simples y sencillos, vivenciados por separado, aunque con relación de totalidad. Por eso una destreza, o un gesto total, o incluso un deporte, requieren ser descompuestos en temas y subtemas y al final, como juntando partes de un rompecabezas, se obtiene la figura, el esquema o modelo final.

Esta visión del aprender y el enseñar otorga al maestro funciones específicas como proveedor de experiencias y verificador de conductas. Sin embargo, algunas funciones son transferibles al estudiante, en especial las consideradas de menor trascendencia, como iniciar el calentamiento de la clase, distribuir y recoger el material, o la demostración física de una destreza, previa o posterior a la explicación verbal del modelo. Las funciones no transferibles se relacionan con el saber cómo en cuanto representa la lógica del proceso de ejecución, las condiciones de ejecución y los criterios de validación. En consecuencia, la enseñanza del maestro se centra en lo que debe ser transmitido, que es a la vez lo que debe ser aprendido por el estudiante y sobre las relaciones de poder que median en el aprendizaje expresadas en un modelo vertical, e interiorizadas de conformidad con el modelo de enseñanza.

Desde la forma en que se enseña se infiere que lo que debe ser aprendido es un saber cómo hacer, un modelo o técnica de movimiento, que además de ser objetivado es simbolizado, y se constituye en el ideal de los propósitos de la formación no sólo física, sino moral, cognitiva, social, afectiva y estética. Los atributos de hombre y movimiento, aunque no son comunicados, ni verbalizados por el docente, hacen parte del sentido de la acción de enseñar en forma global, no consciente, como parte de su teoría general de la educación. Puede pensarse que existe un proceso comunicativo tácito, expresado en la interacción, en la vivencia, la sensibilización y la experiencia del aprendizaje del saber cómo, que no es percibido conscientemente por el estudiante. Los pen- 
samientos del maestro y su acción se materializan, en ausencia del lenguaje verbal, centrando la atención y la competencia cognitiva del estudiante en los referentes de formación implícitos en el saber cómo.

En síntesis, la interacción del maestro con los estudiantes se encuentra mediada por una serie de saberes, conocimientos, creencias, simbolismos, rituales y rutinas, algunas de las cuales son objeto consciente, directa y claramente expresados, pero otros son indirectamente planteados e incluso a menudo asumidos sin que medie explicación o comprensión alguna sobre ellos y aunque no hacen parte directa de los saberes disciplinares, sí guardan relación en la medida en que el sentido de la disciplina es asumida como medio de formación. De tal forma que el saber cómo, esencia de la disciplina, lleva implícitas otras formas de pensar, referidas a lo epistemológico, al saber cómo y al para qué de esos saberes.

\section{Lo epistemológico sobre el saber cómo}

En el pensamiento del maestro sobre cómo enseñar se hacen visibles algunos referentes que hacen posible interpretar las relaciones sujeto-objeto de su conocimiento. La experiencia sensible es la fuente del conocer donde centra la verdad de sus contenidos y las formas para su enseñanza. La experiencia sensible en el estudiante, concretada en el hacer el modelo de movimiento, se convierte en un modelo positivo, en el cual basta experimentar y vivenciar, de una parte, y observar y analizar, de otra, para que haya la certeza del aprehender.

\section{Pensamientos sobre el para qué}

Es un postulado y una creencia del maestro que el conocimiento en Educación Física debe ser útil para la vida en cualquiera de sus tiempos y dimensiones. Así, el aprehender el saber cómo es un medio para desarrollar el potencial y la condición física y motriz, para la recreación, para la adquisición de valores y actitudes, para el logro y el éxito en el deporte y para apoyar otros saberes, como la lectura y las matemáticas. Asimismo, se piensa que tiene finalidades curativas, preventivas y regulativas, provee salud física y mental, controla instintos, pulsiones y contribuye al orden social, a las buenas relaciones, y al desarrollo de funciones mentales superiores como la argumentación y la deducción, todo esto sin requerir procesos conexos a lo motriz, ya que supone que sus formas de enseñanza lo incorporan de manera concomitante.

En conclusión, puede decirse que el pensamiento del maestro en situaciones de clase se ubica en la corriente empírico-analítica, por cuanto el aprendizaje de una destreza o movimiento pasa por un proceso de descomposición de sus partes constitutivas, tal como lo plantean Locke, Berkeley, Hume y Mill. El origen de todo conocimiento es la experiencia. Se trata de que las "verdades innatas" sean "conceptos de causa y consecuencia que para los empiristas pueden ser descompuestos o reducidos a conceptos más simples que se derivan de la experiencia...". Son conceptos analíticos, mientras, para los racionalistas son conceptos sintéticos (Briones, Icfes, 1996). Sin embargo, el pensamiento del maestro, desde su palabra, pareciera tener un enfoque idealista por cuanto hace una "construcción intelectual... de muchos sucesos concretos individuales arreglados con ciertos puntos de vista" (Max Weber, 1973).

\section{Pensamientos alrededor del concepto de desarrollo}

El ejercicio docente supone el conocimiento y dominio de unos principios científicos y críticos sobre los cuales se estructuran los temas para los diferentes grados escolares; dominio que debe reflejarse en dos momentos fundamentales de la realización de la acción educativa: el primero, el momento de la planeación y el diseño de los planes de área; y el segundo, durante la ejecución de cada una de las sesiones de clase. La acción de planeación se realiza en las reuniones de área, y se registra en un documento que se llama Plan de Área o Proyecto de Aula, en el cual se establecen los ejes temáticos, los objetivos y las metas de la clase, las estrategias y recursos metodológicos, y las formas de evaluación de los procesos académicos. En el área de Educación Física dicha planeación, elaborada de manera individual o colectiva, se hace teniendo en cuenta los niveles de escolaridad preescolar, básica y media y se estructuran a partir del conocimiento del docente sobre los fundamentos que le fueron enseñados en su etapa de formación profesional y siguiendo en particular los aprendizajes surgidos de la experiencia docente en cada nivel.

\section{Consecuencia del}

\section{proceso de formación profesional}

En el discurso, el maestro de Educación Física se siente identificado con aquellos conocimientos disciplinares y pedagógicos que aprendió en su etapa de formación profesional; por ello los temas y actividades enunciados en el plan de área se estructuran siguiendo los principios básicos y más elementales de la psicología evolutiva propuesta por Piaget, que formó parte de los 
programas académicos de la formación de maestros en especial a principio de la década de los años ochenta. Las actividades seleccionadas para la realización de las clases de Educación Física corresponden a la consideración del desarrollo biológico particular de los estudiantes y a una valoración inicial que se realiza a partir de la aplicación de pruebas estandarizadas o test de entrada. Estos instrumentos son considerados muy importantes en el quehacer del maestro y evidencian una clara sobrevivencia del modelo de educación por objetivos con los cuales el maestro de Educación Física se siente todavía muy cómodo, ya que le permiten establecer generalizaciones acerca del proceso de aprendizaje de los estudiantes, impartir conocimientos y exigencias físicas de manera global en el aula de clase, desatendiendo en buena medida las tan mencionadas diferencias individuales.

\section{La experiencia profesional:}

\section{un mecanismo de conocimiento del otro}

La tradición y la experiencia que adquiere el maestro de Educación Física en el ejercicio de la profesión han afianzado sus acciones evaluativas tradicionales y lo han convertido en un experto aplicador de pruebas estandarizadas y en detrimento de una actitud reflexiva que e permita replantearse la forma y estructura de su disciplina y que lo alejan cada vez más del conocimiento científico para elaborar su plan de área. Tradicionalmente, prima en el recurso del maestro su experiencia particular y el conocimiento pragmático que va construyendo de manera espontánea a través del tiempo, y sobre el cual no se realiza ninguna acción evaluativa ni de sistematización. Como resultado, y cada vez con maor énfasis, el maestro actúa sobre la base de un profuno desconocimiento de su saber específico y una pobre asimilación de la corriente psicomotricista de la Educación Física que hace de ésta una práctica pedagógica deshumanizada, limitada y limitante.

Considera el maestro de Educación Física que el aprendizaje motor es muy importante en la composición de los temas de su clase como punto central de u aporte al desarrollo de los estudiantes, y en esa dirección enfatiza sus actividades de manera casi inconsciente hacia las dos áreas que lo constituyen: el control motor y el aprendizaje motor. En consecuencia, y conrencido de su coherencia, el maestro propone, una vez más, evaluación a partir de pruebas físicas, que le permiten observar en cada estudiante su nivel de aprendizaje y dominio. Dichas pruebas consisten básicamente en la repetición de una tarea motriz durante un tiempo determinado o la ejecución de series de determinada cantidad de repeticiones impuestas según su criterio particular. Muy pocas veces el maestro puede expresar, frente a este fenómeno de la evaluación, una explicación teórica que supere la premisa de que la ejecución continua de una acción motriz es la única evidencia del aprendizaje; es decir, en el actuar del maestro dentro de la clase se desconocen los procesos básicos que determinan el comportamiento y la modificación de una conducta motriz, y las variables que determinan la eficacia de los aprendizajes, bajo una perspectiva integral del ser humano que supondría la vinculación de saberes complementarios, como la biología, la neurología y la psicología.

En ese sentido, aunque en el discurso del maestro se incluyen como alternativas metodológicas la resolución de problemas, la participación y el autoaprendizaje, como estrategias que facilitan en los estudiantes mejorar los procesos de auto-conocimiento y de aprendizaje autónomo, en su actuar cotidiano, la clase de Educación Física se reduce a la imitación de gestos motores escolares y deportivos impartidos de manera homogénea al conjunto de los grados escolares, siguiendo la cultura pedagógica y didáctica del condicionamiento operante centrado en la repetición del esquema estímulo-respuesta y determinado de manera unidireccional por el profesor, quien decide cuáles y cuántas ejecuciones deben realizar todos los estudiantes de la clase.

En el mismo sentido, el maestro de Educación Física piensa que tiene una responsabilidad en lo que pudiera denominarse la construcción de la historia motriz de los estudiantes. Por ello demuestra un especial interés en proveerlo grado a grado de las experiencias de movimiento que en su entender responden a las necesidades de adaptación biológica diferentes para los niños y las niñas; en ese sentido, regula en cantidad y calidad el esfuerzo que exige a cada uno y las consecuencias que su no realización les traerá a los individuaos en la vida futura. Así pues, el proceso de aprendizaje de Educación Física para la vida se ve permeado y obstaculizado por la necesidad de responder a los cánones corporales impuestos por la sociedad actual; es decir, las experiencias corporales propias del aprendizaje, en su totalidad, las encamina el maestro hacia la búsqueda del ideal de las figuras femenina y masculina, pasando por encima de diferencias individuales, no de género, y de los propósitos legítimos de la Educación Física.

En todo caso, existe una diferencia significativa entre lo que expresa el maestro en su discurso acerca del desa- 
rrollo, la importancia de la realización de la actividad física, la calidad y cantidad de experiencias ofrecidas a los estudiantes y el actuar del maestro centrado en la realización mecánica de ejercicio físico, asociado a la ejecución deportiva y a la búsqueda de la formación de cuerpos esbeltos y fuertes. En la realización de las clases no se hacen evidentes las nuevas tendencias metodológicas de la Educación Física que muy enfáticamente parece haber involucrado en su discurso.

\section{Pensamiento sobre las relaciones maestro-estudiante}

\section{Maestro: autoridad y control}

La clase de Educación Física, en tanto espacio escolar, implica un complejo sistema de relaciones que ocurren en medio de una serie de diferencias de tipo sociocultural, de visión de mundo, éticas y morales, entre el maestro y el estudiante, mediadas por el conocimiento específico que se imparte, representado por los contenidos propios de la Educación Física. De tal manera, analizar el discurso del docente en busca de significados, funciones e intenciones que expresen la realidad de la clase significa comprender las relaciones que suceden en un triángulo cuyos vértices son: el maestro, el estudiante y el conocimiento.

Por tanto, es evidente en el discurso del maestro de Educación Física que él encuentra una conexión muy especial entre el saber específico de su asignatura y la posibilidad de proponer formas de relación más humanizantes y cercanas con el estudiante dentro y fuera de la clase, porque está seguro de que el lenguaje de la actividad física, el juego, el deporte y la calidad de vida, que constituyen la esencia de su clase, lo proveen de un estatus diferente del de todos los maestros de la institución educativa $\mathrm{y}$, por supuesto, en la sociedad en general (véase sección Finalidades) que le permite ser escuchado mucho más que cualquier otro docente, y ser considerado líder dentro de la institución educativa.

Así, en el pensamiento del maestro coexisten de manera natural diversas ideas acerca de cómo deben ser las relaciones en la clase y en la institución educativa. De un lado, se reconoce a sí mismo como autoridad única para ejercer poder dentro y fuera de la clase, gracias a la superioridad que le otorga el cargo y al dominio de su saber específico; y de otro, siente que puede y debe proponer a los estudiantes una relación de amistad, de apoyo y solidaridad a partir de la comprensión de las realidades particulares de los estudiantes y como consecuencia de la interacción que se genera en la dinámica de la realización de la clase relacionadas con la actividad física, el deporte y el juego dentro de la clase.

\section{Autoridad derivada del hacer}

Esta primera forma de relación maestro-estudiante es la consecuencia de la tradición educativa que reza: "Quien enseña, manda y quien sabe, decide". En este caso, el maestro elige para su discurso frente a los estudiantes un tono de autoridad indiscutible que utiliza para organizar tanto las actividades de la clase, como aquellas otras que se derivan de la acción educativa dentro de la escuela. En la medida en que él se reconoce como un modelo a seguir, no permite la discusión sobre sus propuestas de clase ni sobre los valores que quiere inculcar en sus estudiantes. Él sabe, gracias a su formación y principios, aquello que los niños y jóvenes necesitan para alcanzar un nivel de formación adecuado para la vida en sociedad.

En este sentido, el maestro emplea un recurso discursivo que consiste en la presentación positiva de sus creencias y pensamientos a manera de normas y condiciones para la realización de la clase, y las impone como formas únicas de relación, que espera sean reconocidas y obedecidas por el estudiante, pues en su imaginario dicho sistema de relaciones proporciona a los estudiantes los elementos necesarios para su formación integral. Con el mismo énfasis y de manera casi simultánea dentro de la clase, refuerza su autoridad presentando de manera negativa los valores de comportamiento que rigen las relaciones entre los estudiantes.

El maestro de Educación Física justifica el ejercicio de su autoridad sobre el estudiante, tanto en la imposición de los temas como de las normas para la realización de la clase, porque, en primer lugar, no lo considera sujeto con capacidad para decidir sobre lo que conviene a su proceso de formación; y en segundo lugar, porque existe la tendencia generalizada a caracterizar las actuaciones "erróneas" de los estudiantes como parte de las fallas de su ser individual -no de su hacer-y las de los maestros a partir de la circunstancialidad de los sucesos de la clase, es decir, fallas de su hacer. Piensa el maestro que el estudiante actúa fuera de la norma, porque es un sujeto mal educado, vacío de valores, necio, malcriado, desordenado e incapaz; por consiguiente, necesita de un maestro que lo obligue a superar sus deficiencias y carencias y que le enseñe el camino de la transformación dentro del imperante sistema de valores sociales. Entre 
tanto, el maestro ejerce su poder, incluso más allá de los límites de su función, obligado por las circunstancias, y amparado en el cumplimiento de sus funciones educativas. Debido a ello, se siente con autoridad para utilizar los medios y alternativas que a su juicio le permitirán transformar la conducta de sus estudiantes; por eso puede gritar, herir, amenazar, marginar e ignorar a sus estudiantes como último recurso dentro de su labor educativa.

Esta relación de autoridad ejercida por el maestro es aceptada por el conjunto de los estudiantes y reconocida por la comunidad educativa como parte del deber ser del maestro, por cuanto existe la creencia generalizada de que dichas actuaciones responden a la necesidad de ajustar la conducta de los niños y jóvenes a las normas establecidas por el maestro para actuar dentro de la clase y, por supuesto, fuera de ella. De la misma manera la exigencia de la aceptación silenciosa de las normas impuestas por el docente dentro de su clase corresponde a la comprensión que el maestro tiene del deber ser del estudiante, pues es allí donde se concreta el fin último de la educación: la inclusión de los individuos en la sociedad. El maestro actúa con la certeza de que dichas estrategias autoritarias son necesarias para cumplir con su misión de incorporar a la sociedad una juventud dotada de valores como el respeto y la obediencia acrítica de las normas en un contexto en el que la desigualdad y la injusticia social llevan a los estudiantes por el camino de la desobediencia, la rebeldía y la búsqueda de nuevas alternativas de relación con el entorno.

Estas formas discursivas centradas en el ejercicio, y en ocasiones abuso, de la autoridad son elaboradas por el maestro como respuesta a las necesidades de la sociedad y de la institución, y están especialmente dirigidas a los estudiantes considerados problemáticos, de acuerdo con la valoración de su comportamiento dentro de la clase, como por la consideración que el maestro hace acerca del tipo de incorporación que el joven proyecta dentro de la sociedad fuera de la escuela. En este caso, el maestro piensa que es absolutamente necesario imponer sus condiciones, ya que una vez aprendidas las normas escolares, para el individuo será más fácil aprender e incorporar como forma de vida las normas y leyes de la sociedad. Paradójicamente, con los estudiantes que no tienen problemas para ajustarse a su sistema de normas, condiciones y valores, el maestro construye otro tipo de relación, en apariencia más flexible y cordial, que en el fondo, también depende de su reconocimiento como eje central de la acción educativa y de autoridad escolar.

\section{Estrategias verbales para imponer la autoridad dentro y fuera de la clase}

Algunos maestros buscan afectar el comportamiento de los estudiantes a partir del uso de estrategias discursivas verbales y no verbales que suavizan ante el estudiante el ejercicio del poder y en la mayoría de los casos ocultan las verdaderas intenciones del maestro dentro de su clase. En primer lugar, una estrategia, que pudiéramos llamar la forma impersonal, consiste en la preferencia por el uso de la tercera persona pasiva, es decir, su discurso está centrado en lo que se hace como parte del cumplimiento de la función docente, pero que en realidad evita al maestro asumir una total responsabilidad de sus acciones y decisiones. A este grupo de enunciados corresponden expresiones como: el joven fue castigado, hubo que gritarlo y fue sacado de clase, entre otras. De esta manera, el maestro justifica su acción como parte de un deber ser y, sobre todo, evade la responsabilidad sobre sus acciones: no dice yo castigué, grité, saqué de clase.

Una segunda forma muy empleada por el maestro es la forma sarcástica, especialmente utilizada para llamar la atención y corregir a sus estudiantes. Gracias a esta estrategia el profesor no sólo obtiene el poder que otorga la capacidad de burlarse del otro, sino que además goza del reconocimiento y la aprobación del conjunto de los estudiantes, pues casi siempre se emplea como una estrategia para avergonzar en público al estudiante que muestra una conducta calificada por el maestro como inapropiada.

Otra forma de relación que el maestro promueve dentro de su clase podría denominarse de formas ocultas. Consiste en el uso de proposiciones que ocultan el sentido de los enunciados y las decisiones dentro de la clase, en especial en el caso de la extralimitación en la aplicación de sanciones o castigos, cuando el maestro prefiere decir que está formando y no que está castigando, que está impartiendo justicia y equidad o que está garantizando el orden en su clase en cambio de decir que está gritando o marginando.

Por último, dentro de este tipo de relaciones se encuentra el uso de una forma particular de convencimiento: en este caso el maestro recurre al uso de proposiciones cuya intención es hacer sentir al estudiante la necesidad de ser castigado, de ser sometido a la generalidad de norma y, por consiguiente, a la consideración de la aceptación de su comportamiento inadecuado. Esta estrategia consiste en inducir en el estudiante una especie de autorreconocimiento de su condición de inferioridad e inmadurez y autoconvencimiento sobre 
la conveniencia del castigo y la certeza de que es únicamente pensando en su bienestar que el maestro y la institución actúan.

\section{Formas no verbales de imposición de autoridad}

Dentro de las formas no verbales existe una muy particular y sobre la cual el licenciado no se pronuncia. Dicha forma se ha convertido en un instrumento con el cual se imparten órdenes que los estudiantes, con el tiempo, aprenden a comprender e interpretar a satisfacción del docente. Este sistema de comunicación de uso generalizado entre los docentes de Educación Física opera en la dinámica estímulo-respuesta y sirve de apoyo para consolidar la estructura rutinaria de la clase; con él se anuncia el inicio de la clase, el cambio de actividad, la finalización de una tarea, y convoca para los llamados de atención; el silbatazo. El silbato ha venido remplazando la expresión verbal del maestro, convirtiéndose en un elemento primordial para la realización de la clase. Aunque no existe claridad en cuanto al origen del uso del silbato como un sustituto de la voz del maestro en la clase de Educación Física, su uso evoca la del sonido de la diana militar, que indicaba la hora de levantarse, la hora de la batalla, el tiempo del ejercicio, o tal vez sea la adecuación de la estructura autoritaria del juzgamiento deportivo, frente al cual el deportista no tiene más recurso que la obediencia incondicional.

Otras formas no verbales para impartir autoridad son empleadas por el maestro de manera consciente: una de ellas es la intimidación. La mirada intimidante del maestro demuestra una vez más que es considerado como un ser superior dentro de la escuela; la mirada que vigila y puede castigar en caso de observar una conducta inadecuada, mirada que no necesita explicación, pues ya todos deben saber por qué el maestro mira fijamente a un estudiante y, sobre todo, todos deben saber qué hacer cuando el maestro mira fijamente a un estudiante o a un grupo de estudiantes.

A este grupo corresponde una forma particular de forzar cambios de comportamiento en los individuos que muestran mayor dificultad para aceptar las normas: ignorar al estudiante. Esta forma consiste en desconocer al estudiante como participante de la clase, no se le determina como sujeto interlocutor del maestro ni de la clase. En términos coloquiales, consiste en dejar que el estudiante haga lo que quiera en el tiempo de la clase. Así, el maestro considera, en primer lugar, que da una lección al indisciplinado, pues deja de ser parte del grupo, pierde su reconocimiento como ser humano en el grupo al cual por derecho pertenece $y$, en segundo lugar, el maestro hace a un lado su responsabilidad de formar al individuo, al asegurarle que en adelante tendrá que autorregularse si quiere reintegrarse al grupo. Parece querer decirle: estás solo en tu proceso de formación pues ya no cuentas con mi experiencia ni con mi consejo ni con mi saber para guiar tu vida y garantizar tu formación.

\section{El afecto una forma de relación para quienes acatan las normas}

Coexiste en el pensamiento de algunos maestros de Educación Física la idea sobre la conveniencia de proponer otro tipo de relación con los estudiantes, una relación más humana, menos autoritaria, que permita el acercamiento y la confianza entre docente y estudiante. En este caso, el maestro se reconoce a sí mismo como un sujeto capaz de interpretar la realidad socioeconómica y afectiva de los estudiantes y cree tener las herramientas adecuadas para brindar apoyo moral y ético ante algunas circunstancias adversas en la vida del estudiante. A este tipo de relación el maestro asigna el nombre de relación de amistad, pero, incluso en esta dinámica, el maestro ejerce su poder y autoridad mostrándose como oidor de problemas y dador de consejos, ya que en ninguno de los casos ese compartir realidades ocurre en un doble sentido, pues el maestro desconfía del estudiante como confidente y considera inconveniente compartir con él su vida y sus problemas. Existen además dos condiciones impuestas por el maestro para establecer con un estudiante una relación de amistad de la manera ya descrita: primera, que el estudiante pertenezca al grupo de los jóvenes a quienes él considera juiciosos y obedientes, es decir, aquellos que en realidad lo reconocen como modelo, ejemplo a seguir o sencillamente autoridad incuestionable; y segunda, que a corto plazo el maestro tenga evidencias de que su discurso ha afectado de alguna manera el comportamiento del estudiante.

Es evidente que las relaciones maestro-estudiante, a pesar del surgimiento de nuevas tendencias educativas y de la aplicación de diversos modelos pedagógicos en la escuela, siguen ocurriendo bajo el rigor de la autoridad incuestionable del maestro, que se deriva de una lectura muy superficial del contexto y la realidad del estudiante, de donde se origina una serie de interpretaciones equívocas que pueden caracterizarse como una suerte de malentendido entre unos y otros y que desde siempre ha causado en el maestro la sensación de no ser debidamente escuchado, entendido, obedecido y valorado por sus estudiantes, y en el estudiante, la certeza de 
que su maestro es el enemigo a vencer en la batalla educativa. Algunos maestros, desde su discurso, proponen nuevas estrategias de comunicación, pero en la práctica, la realidad de la vida escolar, el número de estudiantes

\section{Referencias}

Cagigal, J. M. (1979). Cultura intelectual y cultura física. Buenos Aires: Kapeluz, 84-85.

Porlan, Rafael y Rivero, Ana. (1998). El conocimiento de los profesores. s. l.: Diada Editora, pp. 13-15.

Tezanos, Araceli de (1988). Una etnografía de la etnografía. Antropos, p. 26.

Unesco. (1996). La Educación encierra un tesoro. Informe de la Comisión Internacional sobre la Educación para el siglo XXI. Madrid: Santillana, pp. 96-106.

Unesco. (1996). "La Educación un tesoro". Informe a la Unesco, presentado por la comisión internacional sobre educación para el siglo XXI. Madrid: Santillana, 95 y 96. en cada grupo, su realidad social, económica y cultural ganan la batalla y de nuevo afloran en la escuela y en la clase las viejas prácticas autoritarias del maestro como eje central del proceso de formación de los estudiantes.

Universidad Pedagógica Nacional. (2002). Documentos Pedagógicos, No. 9. Lineamientos Teóricos de la práctica educativa para los proyectos curriculares. Bogotá: Universidad Pedagógica Nacional. 2002, pp.12,18, 20.

Villar, Luis Miguel. (2001). Pensamientos de los profesores. En: Perafán, Andrés y Adúriz-Bravo, Agustín (Comp.). Pensamiento y conocimiento de los profesores. Debate y perspectivas internacionales. Bogotá: Universidad Pedagógica Nacional, pp. 33-45. 